\title{
Trasplante de médula ósea en pacientes con inmunodefi- ciencia común variable, ¿es una opción terapéutica?
}

\author{
Julio César Cambray-Gutiérrez, ${ }^{1}$ Diana Andrea Herrera-Sánchez, ${ }^{1}$ Patricia López-Pérez, ${ }^{1}$ \\ Aurora Chávez-García, ${ }^{1}$ Marco Antonio Yamazaki-Nakashimada ${ }^{2}$
}

\begin{abstract}
Background: Patients with common variable immunodeficiency show higher incidence of sinopulmonary and gastrointestinal infections, as well as lymphoproliferative and autoimmune diseases. The treatment of choice is replacement therapy with human gamma-globulin. Hematopoietic stem cell transplantation is a non-conventional therapeutic modality.

Clinical case: Twenty-six-year old woman with no family or hereditary history of primary immune deficiencies or consanguinity, with repeated episodes of otitis, sinusitis, gastroenteritis and bronchitis since childhood. At adolescence, she was diagnosed with common variable immunodeficiency; she was prescribed intravenous gamma-globulin, broad-spectrum antimicrobials and macrolides. At 22 years of age, she underwent hematopoietic stem cell transplantation owing to continued severe infections. At 4 months, post-transplantation she was diagnosed with hypothyroidism and ovarian insufficiency. During the following 3 years, she had no infections, but at 25 years of age she had immune thrombocytopenic purpura diagnosed, which persists together with Raynaud's disease and upper respiratory tract persistent infections. At the moment of this report she is being treated with intravenous gamma-globulin and receiving prophylaxis with clarithromycin, without steroids or danazol. Conclusions: Given the high rate of morbidity and mortality associated and immune reconstitution failure, hematopoietic stem cell transplantation should be carefully evaluated in patients with treatment-unresponsive infections or lymphoproliferative disorders.
\end{abstract}

Keywords: Common variable immunodeficiency; Human gamma globulin; Hematopoietic progenitor cell transplant

Este artículo debe citarse como: Cambray-Gutiérrez JC, Herrera-Sánchez DA, López-Pérez P, Chávez-García A, Yamazaki-Nakashimada MA. Trasplante de médula ósea en pacientes con inmunodeficiencia común variable, ¿es una opción terapéutica? Rev Alerg Mex. 2017;64(1):121-125

${ }^{1}$ Instituto Mexicano del Seguro Social, Centro Médico Nacional Siglo XXI, Hospital de Especialidades, Servicio de Alergia e Inmunología Clínica. Ciudad de México, México

${ }^{2}$ Secretaría de Salud, Instituto Nacional de Pediatría, Servicio de Inmunología. Ciudad de México, México
Correspondencia: Julio César Cambray-Gutiérrez. jcesar_963@hotmail.com

Recibido: 2016-02-22

Aceptado: 2016-05-03

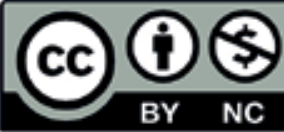




\section{Resumen}

Antecedentes: Los pacientes con inmunodeficiencia común variable presentan mayor incidencia de infecciones sinopulmonares y gastrointestinales, así como de enfermedades linfoproliferativas y autoinmunes. El tratamiento de elección es el reemplazo con gammaglobulina humana. El trasplante de células progenitoras hematopoyéticas es una modalidad terapéutica no convencional.

Caso clínico: Mujer de 26 años de edad sin antecedentes heredofamiliares de inmunodeficiencias primarias ni consanguinidad, con procesos repetidos de otitis, sinusitis, gastroenteritis y bronquitis desde la infancia. En la adolescencia fue diagnosticada con inmunodeficiencia común variable; se le prescribió gammaglobulina intravenosa, antimicrobianos de amplio espectro y macrólidos. A los 22 años se le realizó trasplante de células progenitoras hematopoyéticas por continuar con infecciones severas. A los 4 meses del trasplante se le diagnosticó hipotiroidismo e insuficiencia ovárica. Durante los siguientes 3 años no presentó infecciones, pero a los 25 años manifestó púrpura trombocitopénica inmune, que persistía al momento de este informe con enfermedad de Raynaud e infecciones reincidentes de vías respiratorias altas. Es tratada con gammaglobulina intravenosa y profilaxis con claritromicina, sin esteroides ni danazol.

Conclusiones: Dada la alta tasa de morbimortalidad asociada y falla en la reconstitución inmunológica, el trasplante de células progenitoras hematopoyéticas deberá ser cuidadosamente evaluado en pacientes con infecciones sin respuesta al tratamiento o con enfermedades linfoproliferativas.

Palabras clave: Inmunodeficiencia común variable; Gammaglobulina humana; Trasplante de células progenitoras hematopoyéticas

\author{
Abreviaturas y siglas \\ $\mathrm{EICH}$, enfermedad injerto contra huésped \\ IDCV, inmunodeficiencia común variable
}

IgIV, gammaglobulina intravenosa

$\mathrm{TACl}$, ligando de ciclofilina modulado por calcio

$\mathrm{TCPH}$, trasplante de células progenitoras hematopoyéticas

\section{Antecedentes}

La inmunodeficiencia común variable (IDCV) es la inmunodeficiencia primaria que se diagnostica con mayor frecuencia, con una incidencia entre 1:10 000 y 1:50 000. ${ }^{1}$ Se caracteriza por hipogammaglobulinemia con disminución de 2 desviaciones estándar de IgG e IgA o IgM, además de defectos en la producción de anticuerpos específicos y ausencia de isohemaglutininas. $^{1}$

Los pacientes que padecen IDCV presentan infecciones bacterianas recurrentes, principalmente en los tractos respiratorio y gastrointestinal, causadas comúnmente por microorganismos encapsulados como Haemophilus influenzae y Streptoccocus pneumoniae; Giardia lamblia y colonización por Helicobacter pylori, respectivamente. Las bronquiectasias se registran hasta en $90 \%$ de los pacientes debido a las neumonías de repetición, que incrementan la morbimortalidad en este tipo de enfermos. ${ }^{1,2}$
También se observa mayor incidencia de enfermedades autoinmunes (principalmente citopenias y enfermedad inflamatoria intestinal), enfermedad granulomatosa que simula sarcoidosis, enfermedad pulmonar intersticial, incidencia 30 a 259 veces mayor de trastornos linfoproliferativos, principalmente linfoma de células $\mathrm{B}$ y de cáncer gástrico, respecto a la población en general. En este tipo de pacientes se ha observado mayor morbimortalidad comparados con quienes solo presentan mayor incidencia de procesos infecciosos. $^{2}$

Respecto a la infección, está indicado el uso de antimicrobianos de amplio espectro y el manejo de las complicaciones específicas. El reemplazo con gammaglobulina humana es el tratamiento de elección de la IDCV, 400 a $800 \mathrm{mg} / \mathrm{kg} /$ dosis vía endovenosa cada 21 a 28 días o $100 \mathrm{mg} / \mathrm{kg} / \mathrm{semanal} \mathrm{vía} \mathrm{subcu-}$ tánea, que ha demostrado disminuir significativamente la frecuencia y severidad de las infecciones, el uso de antibióticos y el número de hospitalizaciones. 
El trasplante de células progenitoras hematopoyéticas (TCPH) para pacientes con IDCV se indica en casos seleccionados con complicaciones hematológicas como leucemia mieloide crónica y linfomas o con procesos infecciosos de repetición resistentes al tratamiento adecuado, sin embargo, se registra alta tasa de morbimortalidad y falla inmunológica. ${ }^{2}$

En nuestro país existen estudios que han evaluado la reconstitución inmunológica en pacientes trasplantados (independientemente de la patología de base), los cuales han demostrado que la cuenta de linfocitos CD3 se recupera 6 a 8 semanas después del trasplante, los linfocitos CD8 después de 4 meses y los CD4 después de 6 meses. Las células natural killer reaparecen durante el primer mes y los linfocitos B retornan a valores normales entre 1 y 2 meses después, no obstante, los niveles de IgG se normalizan hasta transcurridos 8 a 9 meses, los de IgM hasta 9 a 12 meses y los de IgA hasta 2 a 3 años. ${ }^{3}$

A continuación se describe la evolución a largo plazo de una paciente con IDCV con pobre respuesta a la gammaglobulina y a los antimicrobianos, quien fue sometida a TCPH.

\section{Caso clínico}

Mujer de 26 años sin antecedentes heredofamiliares de inmunodeficiencias primarias ni consanguinidad, con procesos infecciosos de repetición en la infancia: anualmente presentaba aproximadamente 3 episodios por año de otitis media aguda y de sinusitis aguda, 5 de gastroenteritis aguda y hasta 20 de bronquitis aguda. A los 15 años de edad se diagnosticaron bronquiectasias como secuela de las infecciones respiratorias recurrentes y a los 19 años se identificaron múltiples adenopatías mediastinales; por biopsia se observó proceso inflamatorio inespecífico, por lo que la paciente fue mantenida bajo vigilancia periódica con estudios de imagen.

Se inició estudio por probable inmunodeficiencia primaria. Los resultados mostraron hipogammaglobulinemia en 2 mediciones: $368 \mathrm{mg} / \mathrm{dL}$ de IgG (valor de referencia de $700-1.700 \mathrm{mg} / \mathrm{dL}$ ), $2 \mathrm{mg} / \mathrm{dL}$ de IgA (valor de referencia de $70-350 \mathrm{mg} / \mathrm{dL}$ ) y $7 \mathrm{mg} / \mathrm{dL}$ de $\mathrm{IgM}$ (valor de referencia de 50-300 mg/dL) en la primera; así como $197 \mathrm{mg} / \mathrm{dL}$ de IgG, $16 \mathrm{mg} / \mathrm{dL}$ de IgA y 124 $\mathrm{mg} / \mathrm{dL}$ de IgM en la segunda; además de isohemaglutininas negativas y pobre respuesta a los polisacáridos. Se determinó que la paciente cumplía los criterios diagnósticos para IDCV, por lo que se inició tratamien- to con $400-800 \mathrm{mg} / \mathrm{kg}$ de gammaglobulina intravenosa (IgIV) cada 21 días, manejo antimicrobiano de amplio espectro y ajuste de acuerdo con los resultados de los cultivos, así como macrólidos profilácticos por las bronquiectasias. A pesar de ello, requirió múltiples hospitalizaciones debido a severas infecciones de vías respiratorias.

Por las repetidas infecciones, la paciente recibió TCPH a los 22 años de edad con compatibilidad HLA 10/10 de sangre periférica y acondicionamiento con busulfán, ciclofosfamida y globulina antitimocito, con profilaxis para enfermedad injerto contra huésped (EICH) con ciclosporina. Durante la fase de acondicionamiento, la paciente requirió múltiples transfusiones de concentrados eritrocitarios y aféresis plaquetarias por citopenias prolongadas.

Durante el seguimiento se registraron $\geq 500 \mathrm{cel} / \mu \mathrm{L}$ de leucocitos en el día $11, \geq 50000 \mathrm{cel} / \mu \mathrm{L}$ de plaquetas en el día 18 y $988 \mathrm{cel} / \mathrm{mm}^{3}$ de linfocitos CD3, $193 \mathrm{cel} / \mathrm{mm}^{3}$ de CD 4 y $773 \mathrm{cel} / \mathrm{mm}^{3}$ de CD8 en el día $83 ; 1 \mathrm{mg} / \mathrm{dL}$ de IgA, $18 \mathrm{mg} / \mathrm{dL}$ de IgM y $1145 \mathrm{mg} / \mathrm{dL}$ de $\mathrm{IgG}$ en el día 90 .

A los 4 meses del TCPH se diagnosticó hipotiroidismo e insuficiencia ovárica secundaria a quimioterapia, por lo que la paciente recibe tratamiento sustitutivo hasta el momento de este informe.

Durante los 3 años siguientes, la paciente se mantuvo asintomática, sin procesos infecciosos, pero a los 25 años de edad presentó petequias y equimosis; el diagnóstico fue púrpura trombocitopénica inmune, motivo por el que se le prescribió $10 \mathrm{mg} /$ día de prednisona y $100 \mathrm{mg}$ de danazol cada 12 horas, con persistencia de trombocitopenia moderada que coexiste con enfermedad de Raynaud, en vigilancia reumatológica.

A los 26 años de edad, la paciente reinició con infecciones de vías respiratorias altas (rinosinusitis crónica con pobre respuesta al tratamiento y 3 episodios de bronquiectasias en un periodo menor de 3 meses). Los exámenes de laboratorio indicaron 315,1 y $25 \mathrm{mg} / \mathrm{dL}$ de IgG, IgA e IgM, respectivamente, con posterior determinación de 300,1 y $28 \mathrm{mg} / \mathrm{dL}$ de $\mathrm{IgG}$, IgA e IgM, respectivamente, resultados que corroboraron falla inmunológica con persistencia de manifestaciones clínicas de IDCV. Al momento de este informe, la paciente solo recibía IgIV cada 21 días y profilaxis con claritromicina y aún presentaba 2 a 3 episodios de rinosinusitis aguda al año y trombocitopenia moderada, sin uso de esteroides ni danazol. 


\section{Discusión}

El diagnóstico de IDCV se basa en los criterios diagnósticos establecidos por la Unión Internacional de la Sociedad de Inmunología: edad mayor de 4 años, disminución de 2 o más desviaciones estándar de IgG, IgA o IgM de acuerdo con la edad del paciente, pobre o ausente respuesta a la vacuna contra neumococo, Haemophilus influenzae y tétanos, bajos niveles o ausencia de isohemaglutininas, historia infecciones recurrentes y la exclusión de otras causas de hipogammaglobulinemia. ${ }^{4,5}$

Además de la disminución de las inmunoglobulinas, los pacientes con IDCV presentan otras alteraciones inmunológicas. Algunos informes han descrito reducción de las células $\mathrm{B}$ de memoria en sangre periférica. Brouet y colaboradores demostraron disminución de $70 \%$ de la población de células $\mathrm{B}$ CD27+. ${ }^{5}$ Se han registrado alteraciones también en los monocitos y células dendríticas, las cuales desempeñan un papel importante en el desarrollo de las células B. ${ }^{6}$ También se ha demostrado defecto en la expresión y función de los receptores tipo Toll y en los pacientes con manifestaciones autoinmunes se ha documentado disminución y desregulación de los linfocitos T reguladores. ${ }^{3}$

La mayoría de los pacientes tiene un número normal de células B en sangre periférica, pero se encuentra disminuido el número de células $\mathrm{B}$ de memoria con cambio de isotipo (IgD-IgM-CD27+), así como defectos de hipermutación somática. En algunos casos se han descrito mutaciones autosómicas recesivas incluyendo iCOS, CD19, receptor del activador de células $\mathrm{B}$, ligando de ciclofilina modulado por calcio (TACI), CD20 y CD81. ${ }^{7}$

El reemplazo con gammaglobulina humana es el tratamiento de elección para pacientes con diagnóstico de IDCV, a dosis de 400-800 mg/kg cada 3 semanas, ${ }^{8}$ que ha demostrado disminuir significativamente la frecuencia y severidad de procesos infecciosos y, por lo tanto, el uso de antibióticos y número de hospitalizaciones. ${ }^{9}$

Existen revisiones sobre el manejo adecuado de las complicaciones y comorbilidades, la más reciente es la realizada en 2013 por el Panel de Expertos, donde se describe que los pacientes con procesos infecciosos sinopulmonares requieren antibióticos e incremento de la dosis de IgIV; en sinusitis crónica que no responde al tratamiento está indicada la cirugía endoscópica de senos paranasales, además del seguimiento con tomografía de alta resolución del tórax, espirometrías y cultivos de esputo; en caso de bronquiectasias por infecciones, antibióticos y tratamiento antimicrobiano profiláctico cuando las bronquiectasias son extensas o existe fibrosis pulmonar, acompañada de insuficiencia respiratoria crónica. ${ }^{10}$ En los trastornos autoinmunes se prescriben esteroides sistémicos y otros inmunosupresores o anticuerpos monoclonales antiCD20 e inhibidores de TNF $\alpha$. En los trastornos linfoproliferativos está indicada la quimioterapia; algunos protocolos avalan el uso de rituximab y el TCPH. En esta revisión se encontró que el TCPH también está indicado en aquellos pacientes con IDCV que presenten procesos infecciosos resistentes al tratamiento indicado. ${ }^{10,11}$

Un estudio multicéntrico retrospectivo evaluó a pacientes con IDCV sometidos a TCPH: de 24 pacientes, en 6 la indicación fue linfoma, en 3 infecciones severas a pesar del tratamiento adecuado y en 15 desrregulación del complejo inmunológico (citopenias resistentes e inflamación granulomatosa con afectación orgánica). Se registró una mortalidad de $50 \%$ a un año por complicaciones propias del TCPH (fiebre neutropénica y EICH). Se valoró la reconstitución inmune a largo plazo de los sobrevivientes, definida como la independencia de tratamiento sustitutivo de inmunoglobulina. De los 12 pacientes, solo 6 pacientes presentaron niveles de IgG, IgM e IgA normales, sin embargo, entre los 464 y 3202 días después, 5 de los 6 pacientes presentaron niveles bajos de inmunoglobulinas séricas e infecciones de vías respiratorias altas de repetición, por lo que necesitaron nuevamente IgIV. De tal forma, solo un paciente presentó resolución de la IDCV, sin que se registrara la evolución más allá de ese periodo. ${ }^{12}$ En otro informe de pacientes sometidos a TCPH -2 por trastornos linfoproliferativos, 2 por citopenias resistentes y otro sin registro de la indicación-, los 2 con trastornos linfoproliferativos presentaron resolución de esa condición, pero en ninguno de la IDCV. ${ }^{13}$

En la paciente con IDCV aquí descrita, el TCPH se indicó por persistencia de procesos infecciosos severos a pesar del tratamiento sustitutivo con gammaglobulina, uso de antimicrobianos de amplio espectro y profilaxis por las bronquiectasias. Presentó citopenias prolongadas, falla ovárica e hipotiroidismo como complicaciones del TCPH, y a los 3 años manifestó fenómenos autoinmunes y nuevamente infecciones del tracto respiratorio. 


\section{Referencias}

1. Aydogan M, Eifan AO, Gocmen I, Ozdemir C, Bahceciler NN, Barlan IB. Clinical and Immunologic features of pediatric patients with common variable immunodeficiency and respiratory complications. J Investig Allergol Clin Immunol. 2008,18(4):260-265. Disponible en: http://www.jiaci.org/summary/vol18issue4-num355

2. Thickett KM, Kumararatne DS, Banerjee AK, Dudley R, Stableforth DE. Common variable immunodeficiency: Respiratory manifestations, pulmonary function and high-resolution CT scan findings. Q J Med. 2002;95:655-662.

3. Gómez-Morales E. Trasplante de médula ósea. Experiencia en México. Gac Med Mex. 2002;138(Supl 1):135-144. Disponible en: http://www.medigraphic.com/pdfs/gaceta/gm-2002/gms021u.pdf

4. Ardeniz O, Başoğlu OK, Günşar F, Unsel M, Bayraktaroğlu S, Mete N, et al. Clinical and immunological analysis of 23 adult patients with common variable immunodeficiency. J Investig Allergol Clin Immunol, 2010;20(3):222-236. Disponible en: http://www.jiaci.org/summary/vol20-issue3-num589

5. Vodjgani M, Aghamohammadi A, Samadi M, Moin M, Hadjati J, Mirahmadian M, et al. Analysis of classswitched memory B cells in patients with common variable immunodeficiency and its clinical implications. J Investig Allergol Clin Immunol. 2007;17(5):321-328. Dispionible en: http://www.jiaci.org/summary/ vol17-issue5-num258

6. Bright P, Grigoriadou S, Kamperidis P, Buckland M, Hickey A, Longhurst HJ. Changes in B cell immunophenotype in common variable immunodeficiency: Cause or effect? Clin Exper Immunol. 2012;171(2):195-200. DOI: http://dx.doi.org/10.1111/cei.12010.

7. Resnick ES, Moshier EL, Godbold JH, Cunningham-Rundles C. Morbidity and mortality in common variable immunodeficiency over 4 decades. Am Soc Hematol. 2011;119(7):1650-1657. DOI: http:// dx.doi.org/10.1182/blood-2011-09-377945

8. Busse PJ, Farzan S, Cunningham-Rundles C. Pulmonary complications of common variable immunodeficiency. Ann Allergy Asthma Immunol. 2007;98:1-8. DOI: http://dx.doi.org/10.1016/S10811206(10)60853-8

9. Spickett G. Current perspectives on common variable immunodeficiency. Clin ExpAllergy. 2001;31(4):536542.

10. Abolhassani H, Sagvand BT, Shokuhfar T, Mirminachi B, Rezaei N, Aghamohammadi A. A review on guidelines for management and treatment of common variable immunodeficiency. Expert Rev Clin Immunol. 2013;9(6):561-574. DOI: http://dx.doi.org/10.1586/eci.13.30

11. Chapel H, Lucas M, Lee M, Bjorkander J, Webster D, Grimbacher B. Common variable immunodeficiency disorders: Division into distinct clinical phenotypes. Blood. 2008;112(2):277-286. DOI: http://dx.doi. org/10.1182/blood-2007-11-124545

12. Wehr C, Gennery AR, Lindemans C, Schulz A, Hoenig M, Marks R. Multicenter experience in hematopoietic stem cell transplantation for serious complications of common variable immunodeficiency. J Allergy Clin Immunol. 2015;135(4): 988.e6-997.e6. DOI: http://dx.doi.org/10.1016/j.jaci.2014.11.029

13. Rizzi M, Neumann C, Fielding AK, Marks R, Goldacker S, Thaventhiran J, et al. Outcome of allogeneic stem cell transplantation in adults with common variable immunodeficiency. J Allergy Clin Immunol. 2011;128(6):1371.e2-1374.e2. DOI: http://dx.doi.org/10.1016/j.jaci.2011.07.055 